\title{
Towards Relative Permeability Measurements in Tight Gas Formations
}

\author{
Denis Dzhafarov ${ }^{1}$, and Benjamin Nicot $^{1 *}$ \\ ${ }^{1}$ Total SA, 64018 Avenue Larribau PAU cedex, France
}

\begin{abstract}
Relative permeability is a concept used to convey the reduction in flow capability due to the presence of multiple fluids. Relative permeability governs the multiphase flow, therefore it has a significant importance in understanding the reservoir behavior. These parameters are routinely measured on conventional rocks, however their measurement becomes quite challenging for low permeability rocks such as tight gas formations.

This study demonstrates a methodology for relative permeability measurements on tight gas samples. The gas permeability has been measured by the Step Decay method and two different techniques have been used to vary the saturations: steady state flooding and vapor desorption.

Series of steady-state gas/water simultaneous injection have been performed on a tight gas sample. After stabilization at each injection ratio, NMR T2, NMR Saturation profile and low pressure Step Decay gas permeability have been measured. In parallel, progressive desaturation by vapor desorption technique has been performed on twin plugs. After stabilization at each relative humidity level the NMR T2 and Step Decay gas permeability have been measured in order to compare and validate the two approaches.

The techniques were used to gain insight into the tight gas two phase relative permeability of extremely low petrophysical properties $(\mathrm{K}<100 \mathrm{nD}$, phi $<5 \mathrm{pu})$ of tight gas samples of Pyrophyllite outcrop.

The two methods show quite good agreement. Both methods demonstrate significant permeability degradation at water saturation higher than irreducible. NMR T2 measurements for both methods indicates bimodal T2-distributions, and desaturation first occurs on low T2 signal (small pores).

Comparison of humidity drying and steady-state desaturation technique has shown a 12-18 su difference between critical water saturation (Swc) measured in gas/water steady-state injection and irreducible saturation (Swirr) measured by vapor desorption.
\end{abstract}

\section{Introduction}

In tight-gas sandstone, the productivity of a well is sometimes quite different from that of a nearby well. Wells also can be very sensitive to small amounts of water, whether from an aquifer associated with the reservoir, from hydraulic fracturing, or from other completion operations. In tight rocks, in case of small porosity and very small permeability, a significant influence water saturation on gas phase permeability is expected [1].

Relative permeability governs the multiphase flow; therefore, it has a significant importance in understanding the reservoir behavior. These parameters are routinely measured on conventional rocks, however their measurement becomes quite challenging for low permeability rocks such as tight gas formations.

Tight gas rocks generally have various initial water saturation values. Due to tight nature of the rock, a significant transition zone above gas water contact exists. In this case, presence of water can have a big impact on the effective permeability and, therefore, on the wells productivity. Thus, a proper measurement of effective permeability over different partial water saturations is an important subject for the reservoir characterization and production forecast.

\subsection{Literature review and Issues of $\mathrm{Kr}$ tight gas}

Liquid distribution in a tight gas reservoir controls the flow of gas more critically than in conventional systems [2]. Earlier experimental studies [3, 4] on tight gas sandstone show that the relative permeability to gas decreases dramatically as water saturation increases. While this implies that measurements relating to twophase flow in tight sands should be performed under carefully controlled conditions of stress and water saturation, such elaborate experiments are difficult and time-consuming because of the extremely small flow rates involved.

It was indicated $[3,5]$ that in low-permeability reservoir irreducible water saturation and critical water saturation can be dramatically different. "Permeability jail" concept in low-permeability reservoir was introduced, there is a

\footnotetext{
* Corresponding author: benjamin.nicot@total.com
} 
broad range of water saturations in which neither gas nor water can flow.

Convention holds that connate water has little effect on oil or gas permeability because it occupies the smaller pores [1].

In the recent study [2], by use of laboratory scale experiments and modelling, it was shown that capillary driven transport is an important mechanism that helps to redistribute the water within the tight gas rock samples. Strong effect of water dissipation due to capillary suction (during shut-in period) was demonstrated.

Effect of water saturation on the effective permeability to gas has been the subject of numerous experiments $[6,4,3]$. The common laboratory practice is to use evaporation to desaturate samples, effective gas permeability is measured by either the steady-state or the pulse technique at various levels of water saturation established by evaporation.

However, one can argue that the nature of water distribution resulting from evaporation has certain disadvantages of heterogeneity of water distribution and possible salt precipitation of brine.

\section{Samples and Methods}

\subsection{Our methods}

In this study, we demonstrate a methodology for relative permeability measurements on tight gas samples, with particular attention to the monitoring (quality control) of desaturation process, using NMR T2 and NMR saturation profiles measurements. Two different techniques were used to vary the saturations: steady state flooding and vapor desorption. The gas-phase effective permeability at certain water saturation has been measured by the Step Decay method [7].

Steady-state gas/water simultaneous injection has been performed at elevated pressure drop across the sample in order to achieve faster stabilization. After stabilization at each injection ratio, NMR T2, NMR Saturation profile has been measured to control the water saturation homogeneity.

In parallel, progressive desaturation by vapor desorption (humidity drying) technique has been performed on twin plugs. The experimental technique implies the samples saturation equilibration in a series of relative humidity levels. Saturation is then determined gravimetrically and by NMR T2 volume measurements.

After stabilization at each relative humidity level, NMR T2 and Step Decay gas permeability have been measured in order to compare and validate two approaches.
The Step Decay method is an advanced technique allowing the determination of absolute permeability of tight permeability samples $(<0.1 \mathrm{mD})$ that is not possible or very long in time with standard methods under steadystate flow using Darcy's equation The principle of the Pulse Decay method consists in applying a pulse of pressure on one face of the plug and recording the differential pressure $\Delta \mathrm{P}$ calculated from measurements taken at both extremes of the plug. The Step Decay method is based on the application of several consequently increasing pressure pulses [7]. The principal schema of Step Decay experimental set up is shown on Figure 1.

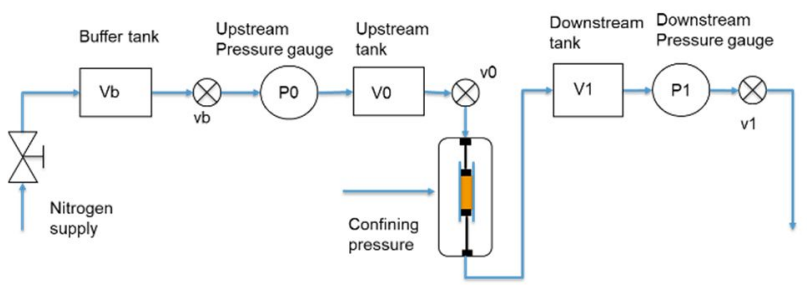

Fig. 1. Schematic of Step Decay experimental device

Step-Decay allows the simultaneous determination of intrinsic permeability (Kl), Klinkenberg coefficient (b) and porosity in one test only, speeding up the laboratory measurement.

Currently, the Step Decay method is used for absolute permeability determination (gas monophasic condition) of permeability of tight rock /gas shale.

Step Decay permeability measurements performed at different gas/water saturations provides gas relative permeability data. In this case, the procedure of experiment is complicated by several steps of sample (de)saturation and have been performed using the following two methods:

- Steady-state gas/water simultaneous injection

- $\quad$ Vapor Desorption (humidity drying)

\subsection{Steady-state gas/water simultaneous injection}

The experiment concerns the progressive desaturation of the rock sample by the steady-state technique

In the steady state test, a sequence of fixed ratios of water/gas fluids is injected through the core sample until saturation and pressure equilibrium is established at each ratio.

Since the permeability involved is extremely low $(<100$ $\mathrm{nD}$,$) , extremely low flow rates are required (<5$ $\mu \mathrm{L} / \mathrm{min}$ ). To ensure such injection requirements an ultra high pressure pump has been employed. 
Humid nitrogen gas and brine are injected to displace gradually the water from the initially fully saturated tight rock sample at constant temperature. Prior to each fractional flow stage, the sample is fully saturated every time by water flooding.

Injection of gas is conducted at constant pressure drop across the core. The water injection is conducted at imposed flow rate on one side of the core (injection). Pressures at the inlet and outlet and the gas flow rate at the outlet of the core sample are monitored with time. The pressure differential to gas and water is assumed to be equal to the measured total pressure drop. Figure 2 shows a schematic of the experimental setup.

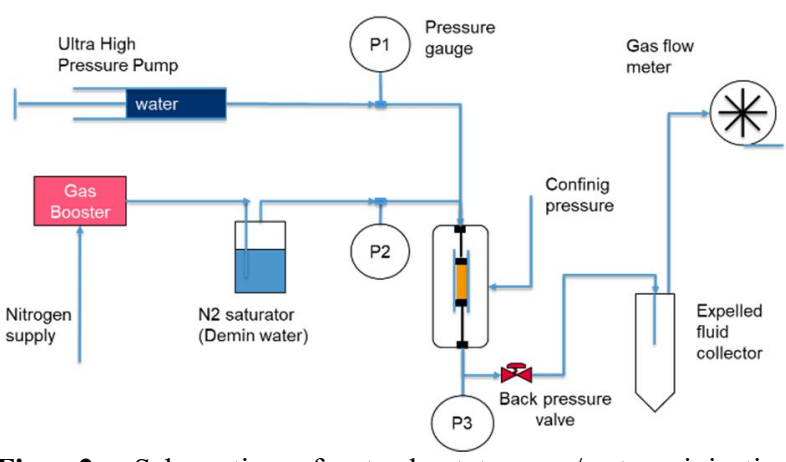

Fig. 2. Schematic of steady-state gas/water injection experimental set up

Each time when stabilization is achieved, injection is stopped and equipment is progressively depressurized. The sample is taken out from the cell and water saturation is determined by gravimetric measurements. NMR T2 and NMR Saturation profiles are also measured to ensure water saturation uniform distribution.

Capillary end effects are a particular problem in gas floods and the potential presence, influence and impact of end effects should be carefully considered when designing a gas displacement relative permeability test program [8]. Steady state water displacing experiments are not generally recommended unless one is aware of the limited saturation range expected from these analyses and of the potential saturation inaccuracies because of disequilibria effects [9]. Thus, the steady-state set up in this case is used only for saturation variation and the gas phase effective permeability is measured by Step Decay method.

\subsection{Vapor Desorption (humidity drying)}

The fundamental principal of vapor desorption method is the observation that the vapor pressure $(\mathrm{Pv})$ above a liquid's curved surface is a function of the liquid surface curvature. Since the capillary pressure $(\mathrm{Pc})$ is a function of the liquid surface curvature as well, both capillary and vapor pressure are the functions of saturation $(\mathrm{Sw}) . \mathrm{Pc}$, therefore, can be calculated from Pv by controlling the relative humidity in the rock pores $[10,11]$.

Initially fully saturated samples are placed in a sealed environment with relative humidity control (Humidity drying)

The test sequence represents a drainage type test (water is displeased by gas) where the wetting phase saturation $\mathrm{Sw}$ (water) is reduced as the humidity of the chamber is reduced. In practical manner, water is removed sequentially from the largest pores down through the smallest pores. Figure 3 schematically illustrates how the water vapor condenses within the pores of different size at different $\mathrm{RH}$ conditions.

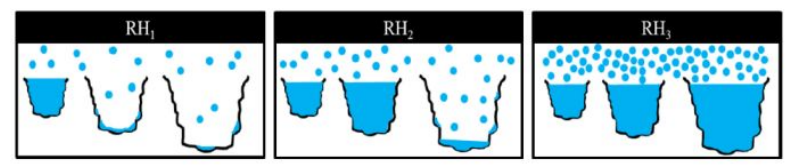

Fig. 3. Vapor desorption/condensation in pores of different size at different relative humidity environments $\mathrm{RH} 1<\mathrm{RH} 2<\mathrm{RH} 3$, after [11]

An electronically controlled humidity chamber is used. It allows investigating the relative humidity from $10 \%$ to $95 \%$ at room temperature. At each humidity level the mass stabilization is achieved and NMR T2 and Step Decay permeability are measured. Water saturation is determined by gravimetric techniques.

The main advantage of this method is the ability to achieve very high capillary pressures (the case for tight gas samples). The salinity increase or probable salt crystallization are the main problems of vapor desorption method that can potentially influence the Pc curve and permeability measurements especially at low saturations. The stabilization time may be several weeks/months.

\subsection{Samples description and preparation}

Identical plugs of Pyrophyllite quarry have been used to perform the test. Pyrophyllite is a homogeneous quarry rock sourced from the United Sates. The samples have preferential water wet wettability. They are mostly composed of clay and consequently has a low permeability (10-200 $\mathrm{nD})$.

A Mercury Injection Capillary Pressure (MICP) and scanning-electron-microscope (SEM) analysis have been carried out on the fragments of the rock (Figures 4 and 5). In Figure 4, the pore throat distribution obtained by MICP shows a continuous distribution of pores approximately in the range between 2 and $30 \mathrm{~nm}$. SEM images analysis shows that two types of pores coexist in this rock: interconnected system of small elongated pores and big pores weakly connected to the main pore system. 

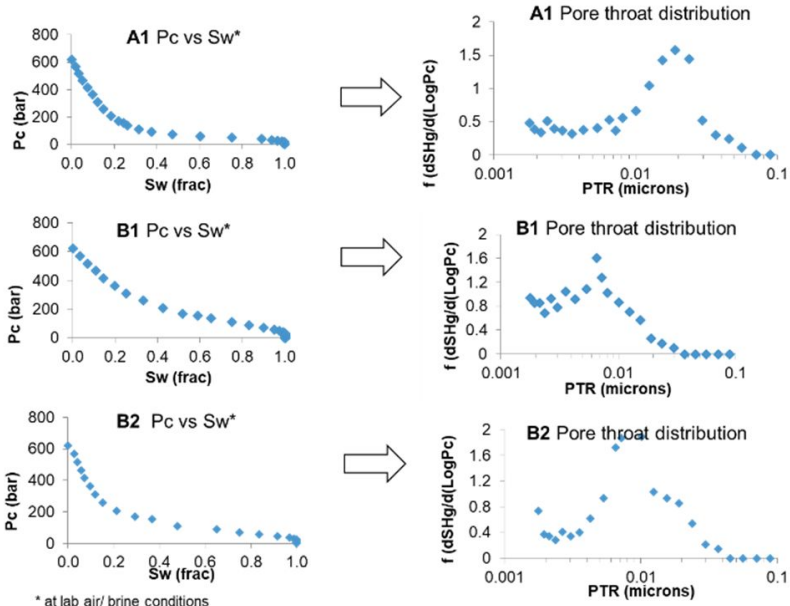

Fig. 4. Capillary pressure $(\mathrm{Pc})$ and Pore throat size distribution (PSD) of Pyrophyllite quarry rock
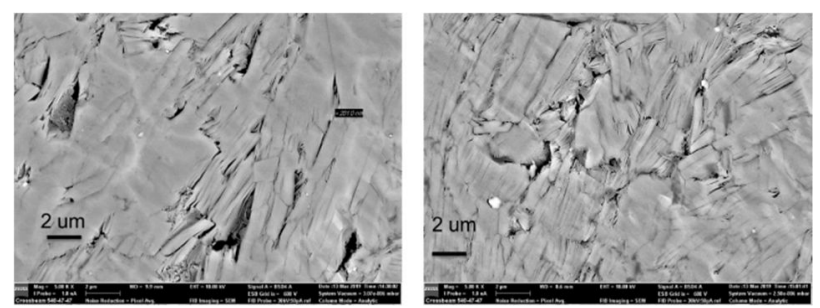

Fig. 5. Scanning scanning-electron-microscope (SEM) typical images of Pyrophyllite quarry rocks

Before starting the experiments the Step Decay permeability $(\mathrm{Kl})$ of each plug were measured at initial "as received" conditions. In this case, the significance of the "as received" condition is that the samples are at the same saturation conditions as in the quarry (atmospheric water) and do not contain hydrocarbons.

Next, the plugs were subjected to Soxhlet cleaning with isopropanol and gentle oven drying at $60{ }^{\circ} \mathrm{C}$ until mass stabilization. Then plugs were fully saturated by $70 \mathrm{~g} / \mathrm{l}$ $\mathrm{NaCl}$ water brine at 500 bar during two weeks, after having created a vacuum over a period of four days.

Total of five pyrophyllite plugs with diameter $23 \mathrm{~mm}$ and height $25 \mathrm{~mm}$ have been prepared:

- 1 plug A1 (Permeability-230 nD and Porosity-5.6 $\mathrm{pu}$ ) has been used for Steady-State flooding experiment

- 4 plugs B1/B2/B3/B4 (Permeability- $80-350 \mathrm{nD}$ and Porosity 4.6 - $6.6 \mathrm{pu}$ ) have been used for Vapor desorption experiment.

\section{Results}

\subsection{Steady-state desaturation experiment results}

The experiment started by only water injection to fully water saturated sample in order to determine brine permeability.
Before each fractional flow stage the sample was fully re-saturated to $100 \% \mathrm{Sw}$. Every fractional flow stage starts by one phase water injection cycle. As soon as the water stabilization is achieved, the gas at higher pressure is applied, while water is continuously injected at the same rate.

Four different gas /water injection ratios have been applied, they are listed in Table 1.

At the final stage, only gas has been injected. For this stage the sample has been again fully re-saturated $(100 \%$ $\mathrm{Sw})$ and the only gas injection has been applied until pressure and gas rate stabilization.

Table 1. Main parameters of gas/water steady-state experiment (sample A1).

\begin{tabular}{|c|c|c|c|c|c|}
\hline TEST & $\begin{array}{c}\text { Water } \\
\text { rate, } \\
\mathbf{m l} / \mathbf{h r}\end{array}$ & $\begin{array}{c}\text { Water } \\
\text { Pres, } \\
\text { bars }\end{array}$ & $\begin{array}{c}\text { Gas } \\
\text { Pres, } \\
\text { bars }\end{array}$ & $\begin{array}{c}\text { NMR } \\
\text { Vol, ml }\end{array}$ & Sw, su \\
\hline $\begin{array}{c}\text { Only } \\
\text { water }\end{array}$ & 0.10 & 100 & - & 0.59 & 100.0 \\
\hline 1 & 0.30 & 260 & 310 & 0.52 & 87.5 \\
\hline 2 & 0.20 & 205 & 310 & 0.45 & 75.8 \\
\hline 3 & 0.13 & 120 & 310 & 0.40 & 68.3 \\
\hline 4 & 0.03 & 53.6 & 310 & 0.34 & 57.3 \\
\hline $\begin{array}{c}\text { Only } \\
\text { Gas }\end{array}$ & - & - & 150 & 0.34 & 56.8 \\
\hline
\end{tabular}

Back pressure for all tests has been set to 32 bars. Net overburden stress has been set to 100 bars.

Critical water saturation (Swc) has been defined at -57 su. High Swc value is expected due to tight nature of the rock (high capillary pressure)

NMR Saturation profile has been performed after each injection ratio: 


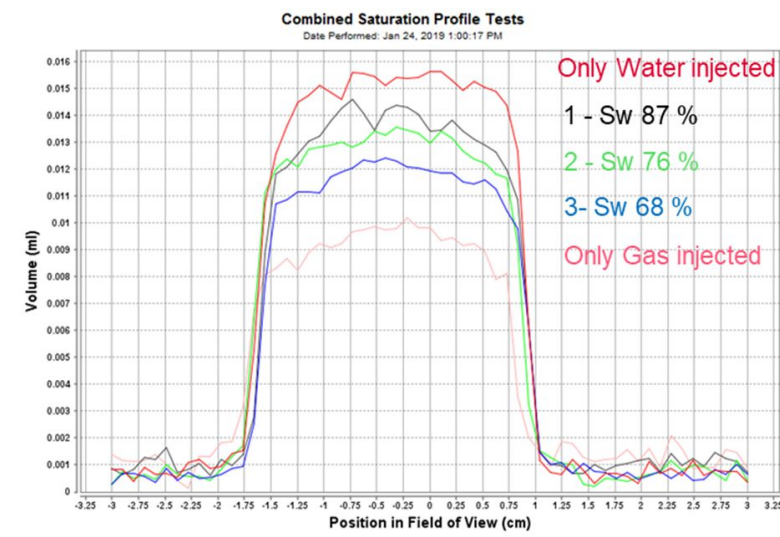

Fig. 6. NMR Saturation profile. Measurements performed after each injection ration at ambient conditions.

Based on Figure 6, saturation profiles appear relatively flat. NMR Saturation profiles do not have any evidence of pronounced capillary end effects. Such saturation behavior could be explained by the combinations of following phenomenon that potentially could influence the saturation distribution and reduce the capillary end effect:

- Water capillary suction. There is around $10 \mathrm{~min}$ time lag between the experiment and NMR saturation measurements, so the water is redistributed by capillarity

- $\quad$ Drying effect

- Effect of gas expansion/compression

The results of Step-Decay gas phase effective permeability measurements over different partial saturation are presented in Figure 7.

It is assumed that use of low pressure step decay method is not influenced by the capillary end effects, as it does not require the water flooding during the experiment.

Water phase effective permeability has been calculated assuming steady-state water flow (see table above). Water phase effective permeability should be treated with caution since it may be biased by saturation disequilibria effects [9]
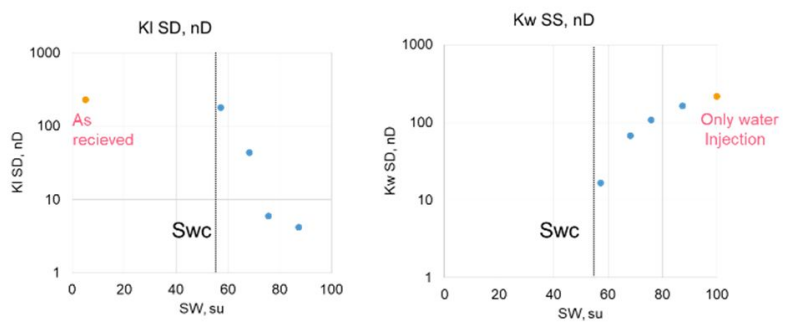

Fig. 7. Gas phase effective permeability measurements by Step-Decay technique. Steady-state gas/water flooding was used to vary the saturation. Water phase effective permeability have been measured by steady-state gas/water simultaneous injection.

Step-Decay gas phase permeability measurements revealed insignificant difference (by factor 1.2-1.5) between the permeability in "as received" conditions and at critical water saturation (Swc). At the same time significant permeability degradation was observed at water saturation being higher than critical. The water phase effective permeability did not show significant decay over Sw decrease (about a decade).

NMR T2 distribution has been measured at each injection ratio. Combined T2 plot is shown on Figure 8.

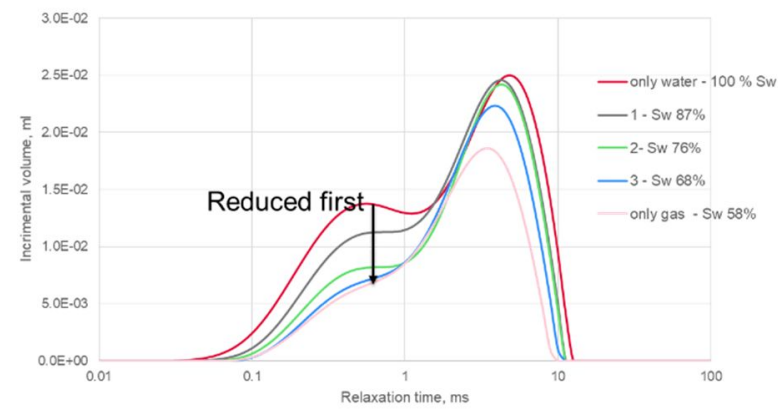

Fig. 8. NMR T2 distribution for different water saturations during steady-state gas/water simultaneous injection

One notes bimodal T2-distributions with higher intensity on second pic. As it can be seen from Figure 8, the desaturation first occurs on low T2 signal. Initially, first decrease of low T2 signal was not expected, as it implies that water desaturation occurs first in low porosity. This could be explained by the assumptions that gas initially flows the path of interconnected system of small elongated pores and desaturates bigger pores at the later stages.

\subsection{Vapor desorption results}

Low permeability sample B1 $(76.4 \mathrm{nD})$ and high permeability samples B2, B3, B4 (230-350 nD) have been used to perform the vapor desorption test.

Initially, fully saturated samples were subjected to four different relative humidity (RH) levels: $90 \%, 85 \%, 80 \%$, $75 \%$. After stabilization at each relative humidity level the NMR T2 and Step Decay gas permeability have been measured in order to compare the two approaches. Stepdecay permeability measurements results are shown on Figure 9.

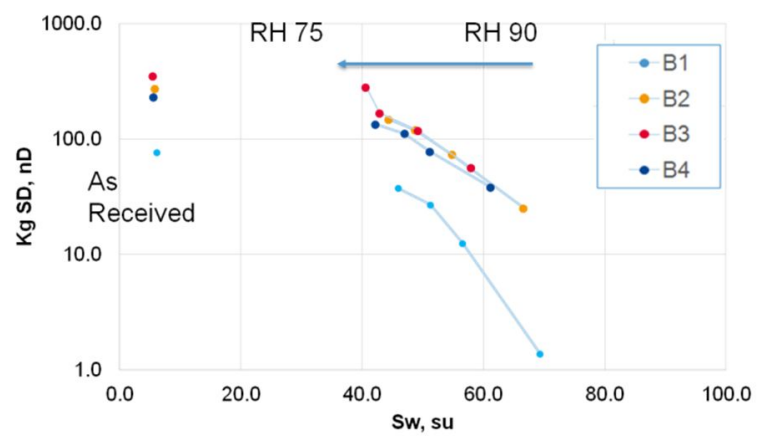

Fig. 9. Gas phase effective permeability measurements by Step-Decay technique. Humidity drying was used to vary the saturation 
Effective gas permeability decay was observed with saturation increase, but less pronounced compared to steady-state desaturation (see Figure 7), since the gas effective permeability high water saturation was not measured (the maximum measured water saturation is limited to RH 90\%).

At Relative Humidity of $75 \%$ saturation levels ranged from $40 \mathrm{su}$ (for B3 sample) to $46 \mathrm{su}$ (for B1 sample). According to the Kelvin equation (Newsham et al 2003), RH $75 \%$ corresponds to approximately 400 bars of capillary pressure in laboratory air/ brine conditions. So, it is assumed that at this pressure irreducible saturation (Swirr) is achieved. Swirr values appear systematically smaller by $12-18$ su compared to Swc measured by steady-state desaturation (see Figure 7).

NMR T2 distribution at each humidity level is shown on Figure 10.
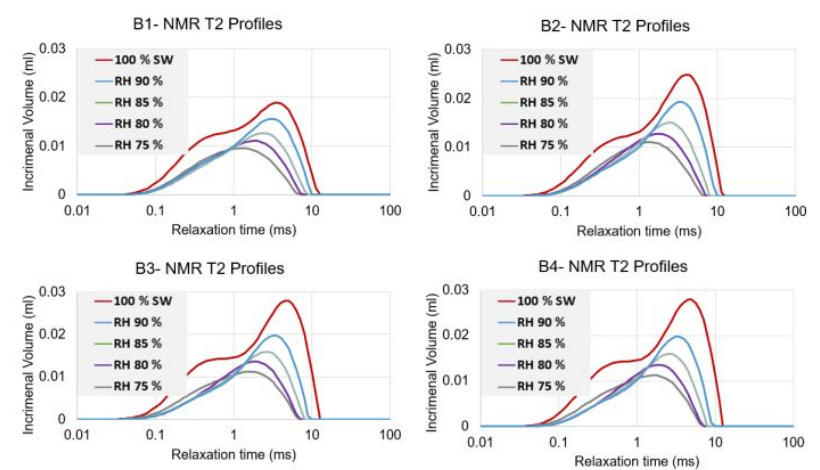

Fig. 10. NMR T2 distribution for different water saturations during vapor desorption desaturation process

NMR T2 distribution during vapor desorption process (Figure 10) confirms that desaturation first occurs on low T2 signal, which was previously observed at steady-state gas/water desaturation experiment (Figure 8).

\section{Discussion}

This study aims at developing new method of tight gas relative permeability measurement and gain insights into the tight flooding specific features. Steady-state regime has been achieved at elevated pressure drop and low injection volumes (see table 1). In this case the direct determination of effective gas phase permeability from experiment is complicated by numerous effects: gas compression/expansion effects, end scale effect, gas viscous instability, etc. On top of that, it is extremely difficult to monitor the downstream fluids production. Thus, the steady-state gas water set-up was used only for the saturation variation. Effective permeability at known saturation was measured by low pressure Step Decay technique. In such way it helps us to avoid many problems related to steady-state relative permeability determination. However, the sample is exposed to the atmosphere which can lead to different undesired influences such as drying and capillary suction. In this case, it is important to control the homogeneity of water saturation. We use NMR Saturation profile measurements to prove the saturation homogeneity.

NMR T2 Monitoring at each saturation level was used to perform analysis of fluids distribution within the pore medium. Despite the fact that the gas is non-wetting phase and it initially flows the path of least resistance through the largest pores, we observe first decrease of low T2 signal at high saturation. Detailed analysis and explanation of this observation is yet to be done.

Comparison of humidity drying and steady-state desaturation technique has shown the difference in critical water saturation (Swc) measured by steady-state gas/water injection and irreducible saturation (Swirr) measured by vapor desorption is 12-18 su. This difference appears mainly in high T2 signal (big pores). Sharp permeability decrease (about a decade) with saturation increase has been observed for both desaturation methods.

\section{Conclusion}

In this study, we demonstrate a methodology for relative permeability measurements on tight gas samples of extremely low petrophysical properties $(\mathrm{K}<100 \mathrm{nD})$ of Pyrophyllite outcrop. We pay attention to the monitoring (quality control) of desaturation process, using NMR T2 and NMR saturation profiles measurements. The gas permeability has been measured by the Step Decay method, and two different techniques have been used to vary the saturations: steady state flooding and vapor desorption. The following conclusion can be made:

- $\quad$ Step-Decay gas phase permeability measurements revealed insignificant difference (by factor 1.2-1.5) between the permeability in "as received" conditions and at critical water saturation (Swc). At the same time significant permeability degradation was observed at water saturation being higher than critical (about a decade).

- NMR T2 measurements indicate bimodal T2distributions and that desaturation first occurs on low T2 signal (small pores). First decrease of low T2 signal was not expected and further detailed analysis of this phenomenon is required

- Comparison of humidity drying and steady-state desaturation technique has shown the difference in critical water saturation (Swc) measured in steady-state and irreducible saturation (Swirr) measured by vapor desorption is $12-18 \mathrm{su}$. This difference appears mainly in high T2 signal (big pores). 


\section{References}

1. S. Motealleh, S. L. Bryant, "Quantitative Mechanism for Permeability Reduction by Small Water Saturation in Tight-Gas Sandstones," SPE Journal, vol. 14, no. 2, pp. 252-258, June 2009 , doi:10.2118/107950-PA

2. D. H. Le, H. N. Hoang, J. Mahadevan, "Gas Recovery From Tight Sands: Impact of Capillarity," SPE Journal, vol. 17, no. 4, pp. 981-991, December 2012, doi:10.2118/119585-PA.

3. K.W. Shanley, R.M. Cluff, J.W. Robinson, "Factors controlling prolific gas production from lowpermeability sandstone reservoirs: Implications for resource assessment, prospect development, and risk analysis," AAPG Bull, vol. 88, pp.1083-1121, 2004, doi:10.1306/03250403051

4. P. Chowdlah, "Influence of Water-Desaturation Technique and Stress on Laboratory Measurement of Hydraulic Properties of Tight Sandstones" SPE Formation Evaluation, vol. 3, no. 4, pp. 679-685, December 1988, doi:10.2118/15210-PA

5. R. M. Cluff, A. P. Byrnes, "Relative Permeability In Tight Gas Sandstone Reservoirs - The "Permeability Jail" Model," presented at the SPWLA 51st Annual Logging Symposium, Perth, Australia, June19-23, 2010, Paper SPWLA-2010-58470

6. J. S. Ward, N. R. Morrow, "Capillary Pressures and Gas Relative Permeabilities of Low-Permeability Sandstone," SPE Formation Evaluation, vol. 2, no.3, pp 345-356, September 1987, doi:10.2118/13882-PA

7. D. Lasseux, Y. Jannot, S. Profice, M. Mallet, G. Hamon, "The 'Step Decay': A New Transient Method for the Simultaneous Determination of Intrinsic Permeability, Klinkenberg Coefficient and Porosity on Very Tight Rocks," presented at the International Symposium of the Society of Core Analysts, Aberdeen, Scotland, 27-30 August, 2012, Paper SCA2012-25

8. C. McPhee, J. Reed, I. Zubizarreta, "Relative Permeability," in Core Analysis: A Best Practice Guide, vol. 64, $1^{\text {st }}$ ed., Amsterdam, Netherlands: Elsevier B.V., 2015, pp. 561-562

9. A. Cense, J. Reed, P. Egermann, "SCAL for gas reservoirs: a contribution for Better experiments", presented at the International Symposium of the Society of Core Analysts, Snowmass, Colorado, USA, 21-26 August, 2016, Paper SCA-2016-23

10. K. E. Newsham, J. A. Rushing, P. M. Lasswell, "Use of Vapor Desorption Data to Characterize High Capillary Pressures in a Basin-Centered Gas Accumulation with Ultra-Low Connate Water Saturations," presented at SPE Annual Technical Conference and Exhibition, Denver, Colorado, 5-8 October, 2003, Paper SPE-84596-MS

11. A. Zolfaghari, H. Dehghanpour, "Pore Size Distribution from Water Adsorption Isotherm," presented at SPE Annual Technical Conference and
Exhibition, Houston, Texas, USA, 28-30 September, 2015, Paper SPE-175155-MS 